\title{
'I'm supposed to see that?' AdChoices Usability in the Mobile Environment
}

\author{
Stacia Garlach \\ University of Hawai'i at Mānoa \\ garlach@hawaii.edu
}

\author{
Daniel D. Suthers \\ University of Hawai'i at Mānoa \\ suthers@hawaii.edu
}

\begin{abstract}
The Digital Advertising Alliance (DAA) recently adapted its self-regulatory notice-and-choice program to the mobile environment by creating a mobile version of its existing opt-out webpage and a new mobile app. Its AdChoices icon remains the primary means on the mobile Web of alerting consumers to the presence of behaviorally-targeted advertising. Previous research has uncovered major usability flaws in the desktop versions, so evaluating the mobile tools is prudent. Mobile devices also present unique usability challenges and afford greater opportunity for tracking consumers. A qualitative study was conducted in which participants $(n=18)$ were given test smartphones and asked to complete three tasks corresponding to the AdChoices icon, webpage, and app. Major usability problems were found that could impact a user's mental model of how these tools work, and the extent to which their privacy is protected. This paper presents findings from the first usability task about the AdChoices icon.
\end{abstract}

\section{Introduction}

The U.S. Federal Trade Commission (FTC) has raised concerns in recent years about how online behavioral advertising (OBA) practices affect consumers' privacy in the mobile environment $[1,2]$. OBA, the practice of sending consumers targeted ads based on data collected by tracking their online activities over time, is now occurring across devices. The online advertising industry is largely selfregulated, but has adjusted its self-regulatory codes in response to privacy concerns raised by the FTC [37]. This study examined new tools created for the mobile environment as part of the industry's noticeand-choice model, in which consumers are notified behaviorally-targeted advertising may be occurring and offered preference-adjusting tools.

Previous studies have found major usability problems with the notice-and-choice model $[8,9]$. It has been widely criticized by consumer advocacy groups [10], academic researchers, legal scholars [11-15], as well as the FTC for not adequately addressing consumers' online privacy concerns. Bennett's [16] helpful discussion of OBA regulation outlines the legal development of the self-regulatory policy regime through 2010. That policy evolves from ongoing FTC public hearings that result in recommendations to the industry, which responds by developing and adjusting its self-regulatory codes.

Hoofnagle et al. [12] argue that advertisers use technology to invalidate consumer choice. The industry claims that OBA is advantageous because people want personalization and customization. However, Turow [17] says that since the industrynot the consumer-is making decisions about customization, consumer "control" is a façade. This type of information asymmetry between a consumer and a company puts the consumer at a disadvantage $[18,19]$. Americans are concerned about data privacy. The 2016 U.S. Consumer Privacy Index [20] found $92 \%$ of Internet users are worried about online privacy, and $74 \%$ said they limited online activity in the past year because of their concerns.

This study contributes to the growing body of literature on the usability and efficacy of selfregulatory privacy choice mechanisms. Participants $(n=18)$ were given test smartphones of the same OS as the phones they owned and asked to complete three usability tasks corresponding to Digital Advertising Alliance (DAA) tools for the mobile environment: AdChoices icon, Consumer Choice mobile webpage, and AppChoices mobile app. Video recordings of participants' hands and screens were used for qualitative analysis in identifying usability problems with the tools. This paper presents results from the AdChoices icon usability task.

\section{Background and related work}

\subsection{Industry self-regulation}

There is little formal U.S. legislation regulating online behavioral advertising. With the exception of a few federal statutes that protect medical, financial, 
and children's data, OBA is subject to industry selfregulation with limited federal oversight. The FTC, in its consumer protection role, has given guidance to the Internet advertising industry based on public hearings $[2,21,22]$ and investigates complaints. The industry has been responsive in hopes of avoiding formal legislation.

The Digital Advertising Alliance (DAA) is the overarching self-regulatory organization for online advertising in the U.S. The Network Advertising Initiative (NAI), with its focus on third-party advertisers, has also been integral in developing the framework. The DAA augmented its 2009 principles [23] with three documents that advise members on the use of multi-site data in 2011 [24], the mobile environment in 2013 [3], and cross-device data use in 2015 [4]. The NAI updated its 2008 self-regulatory code of conduct [25] in 2013 [26] and 2015 [5].

The DAA and NAI codes require giving notice of data collection on website privacy policies. Many studies have shown to these policies to be overly complex, tedious and rarely read [27-30], especially in the mobile environment [31]. Furthermore, though they address cookie-based tracking, they have only recently begun to address other forms of tracking technologies [12], [32-35]. The NAI issued guidance for non-cookie technology in 2015 [7]; the DAA began to address this in April 2017 [36].

The codes also require offering consumers choice to opt out of (or into, depending on information sensitivity) receiving behaviorally-targeted ads. However, even if a consumer chooses to opt out of receiving "interest-based ads," (the industry's term), it does not mean tracking of the consumer will cease.

Besides privacy policies, the OBA disclosure was developed by the industry as a means of notifying consumers about behaviorally-targeted ads. It usually consists of an icon, a tagline, or both, shown on or near an online ad. Not all behaviorally-targeted ads show the disclosure. The icon is very small, typically about 10-20 pixels square. Different icons have been used, but the most prevalent is the Advertising Options, or AdChoices, icon $\gg$ created as part of the DAA and NAI self-regulatory frameworks. A disclosure typically links to information from the entity placing the ad that explains OBA and offers an opt-out. Such opt-outs apply only to that company's ads and are specific to the browser and computer being used. Opt-outs are set by placing third-party cookies on a user's browser; if cookies are cleared, so are the opt-outs.

Curiously, the DAA employs three different websites as part of its self-regulatory program: youradchoices.com, digitaladvertisingalliance.org, and www.aboutads.info, which is where the web- based opt-out tool can be found. During our usability testing, the mobile webpage listed 119-126 participating companies; for the app, there were consistently 32. The NAI lists 105 member companies, 84 of which are also DAA member companies. It has one website, where its opt-out tool resides: www.networkadvertising.org/choices.

Privacy advocates continually raise concerns about OBA practices, and researchers have found the industry's self-regulation measures to be ineffective [8-10], [37] and inadequate in protecting consumer privacy $[38,39]$. Many academic studies have found consumers to have limited understanding of OBA and how they can opt out of receiving it $[8,28,40]$. Even for those who do, opting out of OBA does not necessarily mean they can stop online surveillance and profiling occurring through the industry's data collection practices, which are of greater concern and consequence to consumer privacy [13].

The advertising industry's notice-and-choice model presumes consumers will 1) notice a disclosure, 2) know what it is, 3) know how/be able to click it 4) understand the information provided on linked pages, and 5) figure out how the opt-out mechanism works. Even then, users may think they are making universal and permanent changes, when they may only apply to that site, temporarily. Also, visual presentation of opt-out information is not at all uniform; it varies widely from site to site, as evidenced in this study.

\subsection{Efficacy of OBA choice mechanisms}

Several academic studies have shown the industry's self-regulation efforts to be ineffective at meaningfully addressing consumer privacy. Cranor [37] studied what users understand about OBA, disclosures, and online privacy tools. She and her students found that participants generally lacked awareness about how it works and what tools were available to control preferences. They also evaluated several opt-out tools and found that none of them enabled users to effectively implement their desired tracking preferences. Hastak \& Culnan [9] analyzed the communication efficacy of OBA disclosures. They found that some taglines worked better in conveying the purpose and "clickability" of the disclosures than others, however, they were not the ones being most widely used (e.g., AdChoices). Leon et al. [40] followed up with an online study to test OBA icons, taglines, and landing pages. "AdChoices," the most frequently used tagline in disclosures, was one of the least effective. Users were afraid to click it and were confused about the meaning of opt-out. Ur et al. [41] interviewed non- 
technical Internet users about their attitudes toward OBA. They found that users misinterpreted icons in third-party ad disclosures and did not understand the role of third-party ad networks. Yao et al. [42] did a qualitative study that featured interviews, a card sort and a drawing task to try to better understand people's mental models about OBA. They found four broad "folk models," all either inaccurate or incomplete in representing actual OBA practices.

\subsection{Users' understanding of privacy in the mobile environment}

Because the industry's application of selfregulatory principles to the mobile environment is so recent, little research has been conducted on its implementation. Wobbrock [43] discussed usability and accessibility challenges of mobile devices due to smaller screen, font and button sizes; limited and varying input affordances; and varying use contexts. More specifically, King and Jessen [38] explored personalization and localization privacy concerns that arise when consumers are targeted in the mobile environment. Mobile devices tend to only have one user, unlike desktop devices, so their data is more likely to be linked to an individual. Localization refers to the use of precise geographic location data possible with mobile devices.

Singh et al. [31] tested the readability and comprehension of privacy policies in a mobile environment. They concluded that text-based privacy policies would never be effective in communicating privacy information on mobile devices and recommended simpler, graphical methods. Lin et al. [44] compared users' expectations of what sensitive information an app accesses and why, with what the app actually does. They found participants felt more comfortable when informed why a resource was requesting access for sensitive information like unique device ID, contact list, network location and GPS location. They concluded that informing users of sensitive resource access without explanation did not adequately inform their decision-making.

\subsection{Evaluating AdChoices icon usability}

The usability of the AdChoices icon in the mobile environment will be mapped out using Schaub et al.'s privacy notice design space, then analyzed by applying Norman's interaction design principles.

Schaub et al. [45] developed a useful taxonomy for evaluating types of privacy notice designs based in literature and on expert feedback. They described an effective privacy notice design space in terms of four dimensions: timing, channel, modality and control. Timing addresses when a notice is provided: it can be at setup (helps users decide if tradeoff is acceptable), just-in-time (when data collection is happening), context-dependent (information based context of user or system), periodic (how frequently notice occurs), persistent (ongoing notice when practice is active) or on-demand (user seeks information). The notice's channel can be primary (on the same platform with which the user interacts), secondary (notice is provided elsewhere if system does not afford ability to deliver the primary notice) or public (notice does not target a particular user. A privacy notice's modality describes its interaction modes: visual, auditory, haptic or machine-readable. Control relates to how choices are provided to a user. A blocking notice interrupts the user's action; a user must interact with it in order to continue. Nonblocking notices are less obtrusive and do not deter action. Decoupled notices provide privacy controls in a different location than the notice itself.

Norman [46] outlined seven well-established interaction design principles that can affect a system's usability. Discoverability means a user can easily figure out what something can do and what state the system is in. Feedback is information a device gives a user about the results of actions taken or its current state. A good conceptual model helps a user understand how a system actually works, which can improve its usability. Affordances are possibilities for interaction between user and device. Signifiers communicate how to use a design through affordances, feedback, and constraints. Mapping is the logical relationship between controls and their actions. And constraints limit possible actions, and can be physical, logical, semantic, and/or cultural.

\section{Methodology}

The IRB-approved study consisted of an entry questionnaire, a usability test that employed three tasks corresponding to each of the DAA tools for the mobile environment, and an exit questionnaire. This paper focuses on the usability task with the AdChoices icon.

Study participants $(n=18)$ were recruited by email and word of mouth. The convenience sample included students from two area universities, as well as university foundation co-workers of the one of the researchers. Sessions were conducted in all three places convenient for the participants and lasted approximately one hour.

While the small sample is not generalizable, there were more than enough participants to identify major usability issues. Nielsen [47] found that testing five 
users is typically sufficient to discover most usability problems with a design, and that 15 users can find nearly all the problems. Lewis [48] said that $94 \%$ of problems can be found with four participants in problem discovery usability studies.

This study is unique in that it employed actual phones, rather than simulations, to enhance the ecological validity of the usability testing. Participants were given an iPhone or Android test smartphone according to the model they currently owned. Their activity was recorded on-screen and by videotaping their hands using the phones, to which all participants consented. For iPhone users, the screen of the test smartphone was recorded using QuickTime. Android users' activities were recorded on-screen using the AZ Screen Recorder app, which, unlike QuickTime, could be configured to show where the participant was touching the screen.

\subsection{Usability testing}

After completing an online entry questionnaire, participants were asked to complete three usability tasks that corresponded to each of the three Digital Advertising Alliance tools being tested: the AdChoices icon, Consumer Choice Page for Mobile Web and the AppChoices mobile app. They were asked to think aloud as they went through the tasks. Versions of the usability script were created for Android and iPhone. Both test phones were restored to factory default settings.

iPhone users were given an iPhone 5S running iOS 9.1 restored to factory settings between each participant. Safari Privacy \& Security settings were configured with Do not track toggled to off and Block cookies set to Allow from websites I visit (third-party cookies blocked by default). Before each task, Safari browser cookies were cleared then checked, and the advertising identifier reset. Default settings for iPhone 5S also include Limit Ad Tracking set to off.

Android users were given a Kyocera Hydro Air (C6745) running Android version 5.1.1 (Lollipop). It was also reset to factory defaults between each task. Chrome browsing data was cleared in its privacy settings, then all running apps cleared using the Recents button $(\square)$. In the phone's settings, all apps were closed and memory reset in Usage manager. In Settings > Apps, the action overflow icon ( $:$ ) was used to Reset app preferences. Also within Apps settings, under both Chrome and AppChoices, Clear data, clear cache, force stop were run. Recents was used again to clear all running apps. The final Apps settings adjustment was in Google Settings $>A d s$, where the advertising ID was reset. Then all running apps were cleared again.
One significant difference in privacy defaults between the iOS and Android operating systems is that Safari defaults to block third-party cookies, while Chrome defaults to accept them. This impacted the usability test Task 1 for the AdChoices icon, which will be explained in greater detail in the Results section. Chrome default Privacy settings included Safe browsing turned on and Do Not Track set to off. In Site Settings, pop-ups default to being blocked. Finally, the toggle for opting out of receiving interest-based ads on the Android phone is set to off (so users will receive them by default).

Based on the Leon et al. study [40], participants were given the smartphone with the homepage of a news website active for the first usability task. They were asked to locate the AdChoices icon, then asked what they thought it meant before tapping on it. Depending on what happened when they tapped it, some participants were asked what they thought any options that may have appeared meant. Then they were asked to find where to adjust their ad preference settings and opt out of receiving interest-based ads. That concluded Task 1, and the participant returned the smartphone to be set up for subsequent tasks.

After the usability test, participants completed an exit questionnaire, were debriefed and compensated $\$ 10$ for their time, then offered a list of informational resources about behavioral targeting and tracking.

\section{Results}

Participants $(n=18)$ were smartphone users ranging in age from 18-49. There were ten females and eight males, and education levels ranged from some college to graduate degrees. All participants consented to participate in the study and have it recorded. Nine participants were Android users, running operating systems ranging from 4.44 to 6.0, which was the current operating system at the time of the study. Nine of the participants were iPhone users, running versions of $\mathrm{iOS}$ between 7.1.2 and 9.3.1, which was the latest version at the time. This roughly reflected the breakdown of platform market share among U.S. smartphone subscribers at the time of the study (52.7\% Android and 43.9\% Apple), though iPhones were slightly overrepresented in the sample.

Participants were shown an image of the AdChoices icon and asked to find it on a mobile news webpage. All but two were given the homepage of a local newspaper's website to search for the icon. The remaining two were given a local TV station's news homepage because there were not any ads with icons on the newspaper's homepage at the time of their testing session. Before giving participants a test 
smartphone, the researcher verified that there was at least one icon on the page.

\subsection{Time to find icon}

Typically there were 7-8 display ads on the news site's homepage, which was long for scrolling on a smartphone. Half of the participants $(n=9)$ made it to the bottom of the page at least once before finding the icon. Six participants gave up before finding it, and the researcher assisted by first confirming there was still an icon, and when necessary, informing them it was on the screen.

On average it took participants $1 \mathrm{~min} 6 \mathrm{sec}$ to find the icon; search times ranged from $1 \mathrm{sec}$ to $4 \mathrm{~min}$ $7 \mathrm{sec}$. Two participants accidentally reloaded the page. In one case, the page reload resulted in there no longer being an ad with the icon on the page. The participant gave up $3 \mathrm{~min} 24 \mathrm{sec}$ into the task, when the researcher looked at the phone and confirmed there was no icon present, then clicked into one of the story pages and found two more before returning the phone with an icon showing; the participant saw it within seconds. This case resulted in the longest search time of $4 \mathrm{~min} 7 \mathrm{sec}$; the next longest time was 2 min 53 sec. A third participant had page load problems as a result of a network connectivity error.

\subsection{Icon visibility: size, position, state, color}

According to the DAA's 2013 creative guidelines [49] for the icon and ad marker, the icon should be no smaller than $12 \times 12$ pixels with $10-$ px type $(9.5$ points). Its mobile ad marker guidance [50] recommends a tap area around the marker that is between $20 \times 20$ and $40 \times 40$ pixels.

It is challenging to compare pixel sizes with different devices. The size of pixels is relative to a screen's size and resolution, so the physical size of the icon varies depending on the pixel density of the device on which it appears. In the case of the test phones used in this study, the iPhone 5's pixel density is higher (326 ppi) than that of the Kyocera phone (220 ppi), because it has a higher resolution (640x1136 pixels) on a smaller screen (4"). The Kyocera phone has a 540x960-pixel resolution on a 5" screen. Icons appearing on the test iPhone averaged $23 \times 23$ pixels, while those appearing on the test Kyocera phone averaged 20x20 pixels. The computer was a 13" MacBook Air, which had a 1440x900-pixel resolution and a 131-ppi density, displayed icons at around $15 \times 15$ pixels. See Figure 1 for a comparison, and Figures 2-4 for physical size.

Several participants commented on the icon's size. One mentioned how small it was and that it

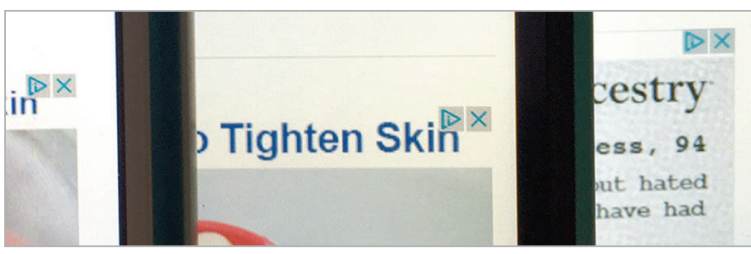

Figure 1: Icons shown on (L-R): iPhone $5 S(23 \times 23 \mathrm{px})$, Kyocera Hydro Air (Android) phone (20x20 px), and MacBook Air (15x15 px).

appeared to be part of the ad. On locating the icon, another participant commented, "It's really small though. I wouldn't have noticed if I didn't see the picture," pointing to the image on the instruction sheet. Other responses were more colorful. One exasperated participant said, "Oh my God, am I blind?" and described the icon as "miniscule" upon finding it. Another participant, who finally spotted the icon after searching for 3 minutes 35 seconds, exclaimed, "Holy s---! I'm supposed to see that? That might as well not even be there!"

In addition to its tiny size, another factor contributing to the obscurity of the icon on the page was its potential state change: sometimes the icon alternated to an " $X$ ". The DAA's mobile ad marker guidance [50] recommends including a persistent close mechanism in the top right corner in keeping with user convention. It even suggests that the ad marker be placed in a different corner, and makes no mention of the animation we encountered.

In total, participants passed the icon 16 times and the X 12 times. Participants passed a state change six times. Of the 19 icons participants found, nearly all were in the top right corner of the ad $(n=16)$; two were bottom left and one was top left. One participant had to find a second icon, which will be explained later. Ten of the found icons alternated with an $X$ in the right corner. One was static in the top left corner. Three ads showed the icon and $\mathrm{X}$ side-by-side with no animation; two sets in the top right corner, one set bottom left. In one case, the ad itself being animated affected the functionality of the icon link: a participant tried tapping the icon as the ad was morphing to another image and it didn't work. She tried again after it stopped and was successful.

The icon's colors and transparency may also contribute to its lack of visibility. DAA's ad marker guidelines specify an icon color palette consisting of a cyan blue (HEX: \#00AECD) for the symbol, which can be used at $30-100 \%$ transparency, on a field of $20 \%$ grey (\#CCCCCC), which can be shown at a $60-$ 90\% transparency. These HEX values were entered into the WebAIM color contrast checker website [51]. The contrast ratio was $1.65: 1$ - at full opacitywhich fails WCAG 2.0 guidelines for contrast ratio 


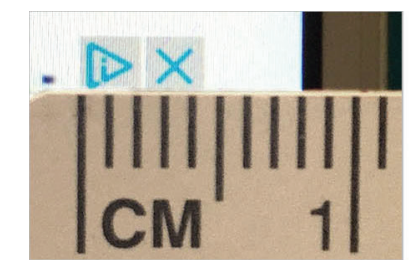

Figure 2: Physical measurement of icon on iPhone. Pixel density: 326 ppi. Icon cropped from screenshot measured $23 \times 23 \mathrm{px}$.

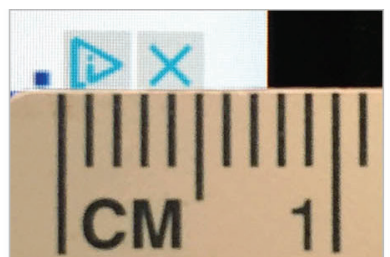

Figure 3: Measurement of icon, $20 \times 20 \mathrm{px}$ on Kyocera, pixel density 220 ppi.

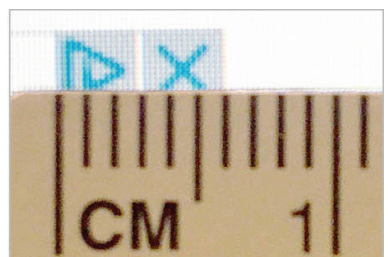

Figure 4: Measurement of icon, $15 \times 15$ px on MacBook, pixel density 131 ppi. requirements for both normal and large sized text at Level AA (4.5:1 for normal sized text; 3:1 for large) and Level AAA (7:1 for normal text; 4.5:1 for large). Any additional transparency would further reduce the contrast. Though the icon is not text, this helps us understand factors that may impact icon legibility. Its size, position within the ad, state, and color can all adversely affect its propensity for being noticed.

\subsection{Icon functionality}

Once participants found the icon, they were asked what they thought it would lead to. Of 16 participants who answered before tapping the icon, 13 (81\%) thought it would lead to the ad, the advertised web page, or more ads. Two participants thought it might lead to information about the ad or why it was there. Another answered, "AdChoices company website; maybe show you a privacy policy or something." One thought it would lead to ad settings.

Two participants mentioned the X. One thought tapping it would close the ad (it did not). Another suggested it was deceptive, in that advertisers may be trying to trick users into thinking they can close the ad, but clicking the $\mathrm{X}$ would actually take them to the ad (also incorrect). In this study we found tapping the $\mathrm{X}$ triggered the same action as tapping the icon.

Two participants tapped the icon before the researcher could ask where they thought it led. In one case, tapping the icon brought up the phrase AdChoices. When asked what it would lead to, the participant said, "Because it says AdChoices, and based on my instructions, I would assume to goes into some kind of privacy control settings." This was the only participant who mentioned privacy settings.

DAA's mobile ad marker guidelines [49] recommend four use cases for what might happen on tapping the icon: 1) link to publisher's choice mechanism or device instructions, 2) open interstitial inside the ad, 3) display additional text next to the icon, or 4) expand the ad with interstitial. Of the 19 ads with found icons, 14 opened an interstitial inside the ad containing additional links when first tapped.
Of those, 13 gave links to three options: AdChoices, Ad covers the page, and Report this ad. The remaining interstitial included a Citi logo and the phrase "Tap to edit advertising preferences" under an $X$. The remaining five ads triggered the phrase "AdChoices" to appear next to the icon.

Eighteen ads had an AdChoices link, most of which led to Google's AdSense Help: About Google Ads page $(n=16)$ on the second tap. One led to a Rocket Fuel opt-out page, and one led to Ghostery opt-out page (which had an Evidon URL and offered one opt-out for Google's DoubleClick ad network).

The Citi ad yielded an unusual result and was the reason an additional ad was selected during this task. Rather than linking to web-based information or an opt-out, the "Tap to edit advertising preferences" link launched iPhone's AppStore and brought up the AppChoices app. This was strange, because the app uses the phone's ad identifier number to set opt-outs, not cookies like the webpage does. This could easily obscure a user's mental model of how opt-outs work.

We tried to find another icon on the page, but the only other one was on another Citi ad, which also led to AppStore. The participant had trouble activating the link: tapping the icon took her to the advertiser's page. She backed up to the homepage, zoomed in on the ad to make the icon larger, tried again, and was taken to the AppStore. We then went into one of the story pages, and we were able to find another icon. This ad it was on was used for the remainder of Task 1 , and is the reason why there was an extra ad.

Other participants also had trouble activating the icon's link. Tapping as the ad was animating or tapping the wrong area caused problems. Some taps brought up interstitials, or went to the ad's website. Even when a first tap triggered the AdChoices text, some had trouble activating the link with a second tap of the icon. Zooming in to maximum view did not help. In one case, the researcher suggested tapping the words, which did activate the link.

On one occasion the icon's tap area collided with the tap areas of both the ad itself and the webpage's back-to-top arrow. Zooming in, a participant clearly 


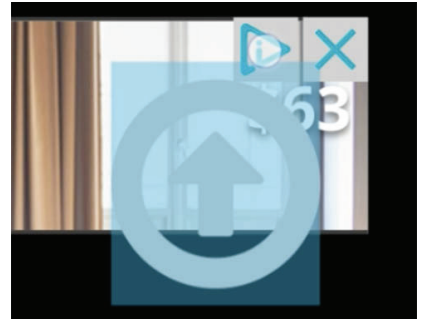

Figure 5: Participant had trouble activating the icon link, despite tapping directly in its center. Icon tap area conflicted with tap areas of back-to-top arrow and ad. tapped in center of icon (Figure 5), but was taken to advertiser's website. She returned to the previous page and scrolled back down the bottom to try again. She then activated the backto-top arrow and became frustrated. After rapidly scrolling back down again and tapping the icon, only to activate the back-to-top button again, the participant exclaimed, "No!" The researcher suggested looking for other icons on the page, which the participant did, but there were none. She then suggested that turning the phone to a horizontal orientation might put the icon in a different position on screen. This worked: the participant scrolled down, zoomed in, and tapped the center of the icon again. The AdChoices interstitial appeared. This process took 1 minute 49 seconds.

\subsection{Adjusting preferences}

Once participants tapped the AdChoices link and arrived at a page that offered them information about opting out, it took them 1 minute 28 seconds on average to figure out how to adjust their preferences $(\min =20 \mathrm{sec}, \max =3 \min 29 \mathrm{sec}$ ). As previously mentioned, most $(n=16)$ were taken to the Google AdSense Help page, which does not contain an optout mechanism. Users must tap an "Ad Settings" link about halfway down the page to reach the opt-out, which is on a different page. This link is in a table under the heading "How you can manage the ads you see," after about 329 words of copy. An anchor link farther up the page under the second section heading can take a user down to the table containing the Ad Settings link. Five participants tapped this link before tapping the Ad Settings link. The remaining 11 scrolled down to it. One gave up before reading the page; the researcher asked him to keep trying.

Once participants tapped the Ad Settings link, they were taken to the "Control your Google ads" page, which contained the opt-out mechanism. They were asked to opt out. This page had two toggle switches that could be used to adjust preferences for interest-based ads: the first was for websites beyond google.com, the second for Google search ads. There was a difference in the default setting on this page between Android and iOS. On the Android phone, both switches defaulted to $\mathrm{ON}$ and were green, corresponding with a section below that explained what $\mathrm{ON}$ meant. The word $\mathrm{ON}$ was green, to the right of the switch, and the word OFF was not present. On the iPhone, the first switch defaulted to a neutral middle position (Figure 6). The switch was gold, the word ON was to the right, OFF was to the left, and both were grey. In the gold field above the first switch, users were asked to set their preferences. The second switch defaulted to ON. Like the Android phone, the switch and word ON were green; the word OFF was not shown. For both platforms, once the switch was turned off, it turned grey and showed the grey word OFF to the right. The word ON did not appear. Five of 16 participants who encountered this page did not scroll down far enough to see the second switch, but the first was consistent with the pattern.

Several participants had problems with these switches. The toggle switch (Figure 6) elicited a swiping motion for six participants. The other 10 tapped the switch and were only successful part of the time. For some, tapping the switch turned it on at first, then they had to tap again to turn it off. Others figured out they had to tap the word to either side of the switch to make it function.

Two participants were taken to a page other than Google. One link led to a RocketFuel privacy policy webpage, which offered an opt-out link after 1,689 words of copy. Once the participant found the link, which said "click here," and

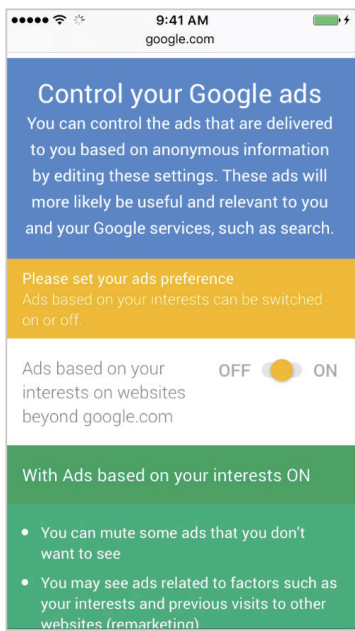

Figure 6: iPhone toggle switch on Google ads preferences page tapped it, she was opted out immediately and returned to the top of the page, where a confirmation message appeared.

The simplest mechanism we encountered in this study was a Ghostery Enterprises (now Evidon) page. It had the shortest, clearest message about opting out preceding its mechanism on the same page:

\section{How Data Powers Your Experience}

You can opt out of any or all of the companies listed below. Opting out does not mean you will stop seeing ads. It means that the company you opt out of will no longer use your data to target ads to you.

The participant who encountered this mechanism responded favorably. She was surprised that the icon would enable her to exercise her preferences, and appreciated learning about it. This tool required 
ticking a checkbox next to companies from which she wanted to opt out (there was only one in the listDoubleClick), then tapping a button that said, "Optout from selected companies." After she ticked the checkbox, she zoomed out to read the information in the right column under the heading, "About interestbased-advertising." Once she read the button and tapped it, the checkbox turned into tiny text that said, "opted out," which she did not notice at first. She tapped the button again, read its text aloud, then noticed the change.

Such different experiences of exercising "choice" can impact a user's mental model of what will protect their privacy. They may think using one of these choice mechanisms precludes them from receiving all behaviorally targeted advertising, which is not the case. Opt-out cookies are needed from all advertising companies that would place ads on a user's device, and only work for that device. Even then, this would only prevent behaviorally-targeted ad placement and not necessarily inhibit the tracking of the consumer. It is worth noting that none of the icons used in this study led to the Consumer Choices Page for Mobile Web, where users can opt out from many companies at once. The icons we tested all led to an opt-out mechanism of one particular ad network, presumably the one placing the ad on which the icon appeared.

\section{Discussion}

Our study showed several design problems that impact the usability, and therefore effectiveness, of AdChoices disclosures in the mobile environment.

\subsection{AdChoices disclosure design}

The Schaub et al. taxonomy considers four dimensions of the privacy notice design space: timing, channel, modality, and control. The timing of the AdChoices disclosure could be considered "just in time," in that it is shown on a behaviorallytargeted ad when it is placed. Since it is provided on the same device with which the user interacts, it is delivered through a primary channel. The modality of the icon is visual, and is sometimes includes a textual notice beside it. In terms of control, the icon is supposed to link to an opt-out choice mechanism. It is a non-blocking notice in that it does not require the user to interact with it. Because of this, it is often ignored. It is usually decoupled from the choice mechanism with a varying amount of information between them.

Despite previous research that found both this icon and the phrase "AdChoices" to be ineffective in communicating about OBA practices and options [9], [40], the industry is committed to using both as the cornerstone of its self-regulatory notice-and-choice program. While the disclosures are being used more consistently than in previous years, there is still a lack of continuity in how they appear and function.

\subsection{Finding the AdChoices icon}

Many participants had trouble locating the AdChoices icon on a mobile news website, despite being shown the icon beforehand. There are several factors that may have contributed to this difficulty.

The arbitrary symbol and its tiny size impede its discoverability, in that if users do not know what it is or cannot see it, they will not know it serves as a gateway to choices regarding OBA.

The color contrast ratio of the icon is quite low, which may be exacerbated by the background on which it appears and varying levels of transparency. This can also compromise the icon's visibility. Another factor that made the icon difficult to find in some instances was a state change of the icon or the ad: several participants passed an $\mathrm{X}$ while searching for the icon. The icon's position on an ad also varied in different cases; it might be found in any of the ad's four corners. Furthermore, at times it was placed in the top right corner and alternated with an $\mathrm{X}$, which emulates the web convention of a window closing mechanism. In that case, the $\mathrm{X}$ is false signifier of a close mechanism that did not exist: tapping it led to the same outcome as tapping the icon. One participant even mentioned this seemed deceptive.

\subsection{Using the AdChoices icon}

Norman [46] says a good conceptual model gives users an accurate idea of how the system actually works, which improves their ability to use it. The icon falls short in this regard. Most users did not understand that the icon was a link separate from the ad, let alone that it would lead to an opt-out mechanism. Tapping the icon led to a variety of outcomes in this study, none of which included linking to the Consumer Choices webpage. Also, it led not only to different types of feedback events on first tap (i.e., AdChoices phrase appears or interstitial with more links appears), but a second tap linked to various opt-out tools for a particular company placing the ad. This lack of consistency can inhibit users' understanding of how OBA disclosures work. A better solution would be for the icon to consistently take the user to the Consumer Choices page, where they can opt out of many companies at once. 


\subsection{Study limitations}

Our study had several limitations. The fact that we used actual phones (rather than simulations, as in other studies) was generally a strength that yielded rich, realistic qualitative data. However, it did present challenges. Participants did not use their own phones, with which they may have been able to interact more easily. There were also technical difficulties: Wi-Fi network interruptions, unexpected alert messages, and accidental page refreshes impacted the consistency of participants' experiences during the study. Using active webpages created challenges for consistency. We could not control for ads with icons. Finally, most of the icons we found led to the About Google Ads webpage. Considering the DAA has more than 300 member companies, there are likely many more scenarios we could have experienced had we encountered ads placed by different networks. The two others we did encounter, RocketFuel and Evidon, offered very different experiences.

\section{Conclusions}

This study sought to explore the usability of the DAA's consumer choice tools for the mobile environment. Consistent with previous studies, major usability problems were found that can negatively impact a user's understanding of the notice-andchoice model that is at the heart of the online advertising industry's self-regulatory efforts, specifically with the use of the AdChoices icon.

Considering the icon was designed by an industry whose expertise lies in creating noticeable messages, it falls short of its efforts to establish notice, let alone make choice evident. And while the DAA website claims its icon appears more than a trillion times monthly, our study corroborates Leon et al. [46] in finding that participants still have a hard time noticing it and generally do not understand what it means if and when they do. More importantly, we found that mobile device affordances and constraints both intensify previously identified usability problems and create a propensity for new ones.

\section{References}

[1] Federal Trade Commission, "Cross-device tracking: An FTC staff report," Staff Report, Jan. 2017.

[2] FTC, "Mobile privacy disclosures: Building trust through transparency," Federal Trade Commission, FTC Staff Report, Feb. 2013.
[3] Digital Advertising Alliance, "Application of selfregulatory principles to the mobile environment." Jul2013.

[4] Digital Advertising Alliance, "Application of the selfregulatory principles of transparency and control to data used across devices." Nov-2015.

[5] Network Advertising Initiative, "2015 Update to the NAI code of conduct." 2015.

[6] Network Advertising Initiative, "2015 Update to the NAI mobile application code.” 2015.

[7] Network Advertising Initiative, "Guidance for NAI members: Use of non-cookie technologies for interest-based advertising consistent with the NAI code of conduct." 18-May-2015.

[8] P. Leon, B. Ur, R. Shay, Y. Wang, R. Balebako, and L. Cranor, "Why Johnny can't opt out: A usability evaluation of tools to limit online behavioral advertising," CHI '12, pp. 589-598, 2012.

[9] M. Hastak and C. Culnan, Future of privacy forum online behavioral advertising "icon" study. 2010.

[10] R. Gellman and P. Dixon, "Many failures: A brief history of privacy self-regulation in the United States," World Privacy Forum, Oct. 2011.

[11] S. Barocas and H. Nissenbaum, "On notice: The trouble with notice and consent," 2009.

[12] C. J. Hoofnagle, A. Soltani, N. Good, D. J. Wambach, and M. D. Ayenson, "Behavioral advertising: The offer you cannot refuse," Harvard Law \& Policy Review, vol. 6, no. 2, pp. 273-296, 2012.

[13] E. A. Brotherton, "Big brother gets a makeover: Behavioral targeting and the third-party doctrine," Emory Law Journal, vol. 61, pp. 555-601, 2012.

[14] D. J. Solove, “'I've got nothing to hide' and other misunderstandings of privacy," San Diego Law Review, vol. 44, no. 4, pp. 745-772, 2007.

[15] H. Nissenbaum, "A contextual approach to privacy online," Daedalus, vol. 140, no. 4, p. 32, 2011.

[16] S. C. Bennett, "Regulating online behavioral advertising," John Marshall Law Review, vol. 44, pp. 899-1107, 2011.

[17] J. Turow, The daily you: How the new advertising industry is defining your identity and your worth. New Haven: Yale University Press, 2011.

[18] Y. Li, "Theories in online information privacy research: A critical review and an integrated framework," Decision Support Systems, vol. 54, no. 1, pp. 471-481, Dec. 2012.

[19] A. J. Johnson, "Public comments on FTC \#228 Project No. P859900: OBA: Moving the discussion forward to possible self-regulatory principles." Federal Trade Commission, 11-Apr-2008.

[20] TRUSTe and National Cyber Security Alliance, 2016 TRUSTe/NCSA consumer privacy infographic - US edition. 2016 
[21] FTC, "Self-regulatory principles for online behavioral advertising," Federal Trade Commission, Washington, DC, Federal Trade Commission Staff Report, 2009.

[22] FTC, "Protecting consumer privacy in an era of rapid change," FTC Report, Mar. 2012.

[23] Digital Advertising Alliance, "Self-regulatory principles for online behavioral advertising." Jul2009.

[24] Digital Advertising Alliance, "Self-regulatory principles for online multi-site data." Nov-2011.

[25] Network Advertising Initiative, "2008 NAI principles: NAI's self-regulatory code of conduct.” 2008.

[26] Network Advertising Initiative, "2013 NAI code of conduct." 2013.

[27] A. M. McDonald and L. F. Cranor, "The cost of reading privacy policies," ISJLP, vol. 4, p. 543, 2009 2008.

[28] L. F. Cranor, "Necessary but not sufficient: Standardized mechanisms for privacy notice and choice," J. on Telecomm. \& High Tech. L., vol. 10, p. 273, 2012.

[29] S. Komanduri, R. Shay, G. Norcie, B. Ur, and L. F. Cranor, "AdChoices? Compliance with online behavioral advertising notice and choice requirements," Carnegie Mellon University, CyLab, Oct. 2011.

[30] K. S. Schwaig, G. C. Kane, and V. C. Storey, "Compliance to the fair information practices: How are the Fortune 500 handling online privacy disclosures?" Information \& Management, vol. 43, no. 7, pp. 805-820, Oct. 2006.

[31] R. I. Singh, M. Sumeeth, and J. Miller, "Evaluating the readability of privacy policies in mobile environments," International Journal of Mobile Human Computer Interaction, vol. 3, no. 1, pp. 5578,2011

[32] J. R. Mayer and J. C. Mitchell, "Third-party web tracking: policy and technology," 2012, pp. 413-427.

[33] G. Acar, C. Eubank, S. Englehardt, M. Juarez, A. Narayanan, and C. Diaz, "The Web never forgets: Persistent tracking mechanisms in the wild," 2014, pp. 674-689.

[34] M. Ayenson, D. J. Wambach, A. Soltani, N. Good, and C. J. Hoofnagle, "Flash cookies and privacy II: Now with HTML5 and ETag respawning," SSRN Electronic Journal, 2011.

[35] A. Kuehn and M. Mueller, "Profiling the profilers: Deep packet inspection and behavioral advertising in Europe and the U.S.," SSRN Electronic Journal, 2012.

[36] Digital Advertising Alliance, "NAI and DAA launch new version of choice tools for interest-based advertising," 04-Apr-2017.
[37] L. F. Cranor, "Can users control online behavioral advertising effectively?" Security \& Privacy, IEEE, vol. 10, no. 2, pp. 93-96, Apr. 2012.

[38] N. J. King and P. W. Jessen, "Profiling the mobile customer - Privacy concerns when behavioural advertisers target mobile phones - Part I," Computer Law \& Security Review, vol. 26, no. 5, pp. 455-478, Sep. 2010.

[39] N. J. King and P. W. Jessen, "Profiling the mobile customer - Is industry self-regulation adequate to protect consumer privacy when behavioural advertisers target mobile phones? - Part II," Computer Law \& Security Review, vol. 26, no. 6, pp. 595-612, Nov. 2010.

[40] P. Leon et al., "What do online behavioral advertising privacy disclosures communicate to users?" WPES '12, pp. 19-30, 2012.

[41] B. Ur, P. Leon, L. Cranor, R. Shay, and Y. Wang, "Smart, useful, scary, creepy: perceptions of online behavioral advertising," SOUPS '12, pp. 1-15, 2012.

[42] Y. Yao, D. Lo Re, and Y. Wang, "Folk models of online behavioral advertising," in Proceedings of the 2017 ACM Conference on Computer Supported Cooperative Work and Social Computing, New York, NY, USA, 2017, pp. 1957-1969.

[43] J. Wobbrock, "The future of mobile device research in HCI," in What is the Next Generation of HumanComputer Interaction? 2006.

[44] J. Lin, S. Amini, J. I. Hong, N. Sadeh, J. Lindqvist, and J. Zhang, "Expectation and purpose: Understanding users' mental models of mobile app privacy through crowdsourcing," in Proceedings of the 2012 ACM Conference on Ubiquitous Computing, 2012, pp. 501-510.

[45] F. Schaub, R. Balebako, A. L. Durity, and L. F. Cranor, "A design space for effective privacy notices," in Eleventh Symposium On Usable Privacy and Security (SOUPS 2015), 2015, pp. 1-17.

[46] D. A. Norman, The design of everyday things: Revised and expanded edition. Basic books, 2013.

[47] J. Nielsen, "Why you only need to test with 5 users," Nielsen Norman Group, 19-Mar-2000. [Online]. Available: https://www.nngroup.com/articles/whyyou-only-need-to-test-with-5-users/.

[48] J. R. Lewis, "Legitimate use of small samples in usability studies: Three examples," IBM, Inc., Technical report, 1991.

[49] Digital Advertising Alliance, "DAA icon ad marker creative guidelines, v. 1.1." 30-Oct-2013.

[50] Digital Advertising Alliance, "DAA ad marker implementation guidelines for mobile - Version 1.0.” Apr-2014.

[51] Utah State University Center for Persons with Disabilities, "WebAIM color contrast checker." [Online]. Available: http://webaim.org/resources/contrastchecker/. 